\title{
SOLVING THE POINT CORRESPONDING PROBLEM BETWEEN MULTI RESOLUTION SATELLITE IMAGES USING A MODIFIED SVD-MATCHING ALGORITHM
}

\author{
Ahmed F. Elaksher ${ }^{1}$ and Abdullatif A. Alharthy ${ }^{2}$ \\ ${ }^{1}$ Faculty of Engineering, Cairo University \\ ${ }^{2}$ Faculty of Engineering, Umm Al-Qura University
}

(Received August 26, 2007 Accepted October 4, 2007)

\begin{abstract}
Satellite images are the main source for different change detection applications. These images are usually taken from different sensors at different times. Hence, the point correspondence is not established. In this paper, the Singular Value Decomposition (SVD) is used to automatically solve the point correspondence problem between satellite images with different attributes. For each pair of the images, a cost matrix is built. The cost of corresponding any two points in a pair of images is computed using different geometric attributes. The SVD for the cost matrix is then used to solve the point correspondence problem. The algorithm is tested on QuickBird, IKONOS, and SPOT images. Results showed that using the traditional Euclidean distance cost matrix is not suitable for remote sensing images. Hence, a modified cost matrix was introduced based on the orientation parameters of the satellite images. The new cost matrix is then computed and its SVD is calculated. Results showed significant improvement in the solution of the point corresponding problem regardless the size or orientation of the satellite images.
\end{abstract}

KEYWORDS: Point Correspondence - Satellite Images Transformation Models- SVD - Matching.

\section{INTRODUCTION}

Recent progress in remote sensing imaging systems started a new mapping era. Images from different satellites, such as; Landsat, SPOT, IRS, IKONOS, QuickBird, etc, are being used in a variety of applications. These applications include; land management and monitoring, planning, development applications, etc. One of the main applications of satellite images is the change detection. The aim of any change detection process is to recognize and identify temporal variations at a given location [7]. This application is implemented using a multi-source, multi-resolution, and temporal satellite images. These images are captured by a variety of imaging satellite systems; hence they differ in their geometric properties, i.e. orientation, resolution, origin. Thus, these images have to be registered before they are employed in any change detection process. Image registration is described as the process of identifying corresponding points in two or more images. Hence, the coordinate of the corresponding points are used to alien these images. The quality of the image registration process affects the results and outcomes of the change detection process dramatically [11]. This process can be accomplished either manually or semi-automatically. In a manual image registration system, the 
operator identifies each point and its correspondences in two or more opened images on the computer screen. However, this process needs an expert operator and consumes time and money. In a semi-automatic system, the operator open each image separately and identify the tie points, then a point corresponding algorithm is used to find matched points.

This paper presents a modified and efficient method to automatically solve the point correspondence problem between a pair of satellite images based on the Scott and Longuet-Higgins algorithm. This method has been used before in close range environment. In its traditional implementation, the method depends on computing the proximity matrix between all point pairs using the Euclidean distance between the points regarding they lay on the same plane. However, this implementation showed poor results. Hence, a modified version was adapted based on the 4 parameter transformation model. The modified version showed reliable and stable results.

The paper is organized as follows. First, a brief summery of recent methods used to solve the point correspondence problem and register satellite images is presented. In the next section, the original Scott and Longuet-Higgins algorithm is introduced. The modification process is then described. Experimental results using SPOT, IKONOS, and QuickBird images are then discussed and analyzed. Conclusions are then stated.

\section{BACKGROUND}

Habib and Al-Ruzouq [4] used the Modified Iterative Hough Transformation (MIHT), presented in [6], to register a dataset of different satellite images. The images include; IKONOS, SPOT-5, Landsat-7, Quickbird, Orbview, and EROS-A1 satellite images. Linear features were used as the primitive registration features. The 2D affine and similarity transformations were used to establish the mathematical relation between the features. The algorithm then generate all possible matching hypotheses between the registration primitives for a given image pair. For each hypothesis, the transformation parameters are computed. Each hypothesis then vote in its corresponding cell in an accumulator array of the transformation parameters. Matching hypothesis that contribute to the peak cell are used to establish the corresponding primitives [5]. Such approach is time consuming and requires intensive effort in selecting the accumulator cell size, searching, optimum sequence for the parameter estimation [1].

Lavigne [10] described an image registration algorithm based on detecting features that have invariant in translation and rotational transformations, invariant in illumination, and identify the ones that remain persistent through scale changes [12]. Classified translation, rotational, illumination, and locally scale-invariant features are then validated through a decimation process. Selected remaining invariant features are expressed as descriptors, which are used to establish feature correspondences. The relationship between descriptor sets is then established by estimation of an affine transformation model, used subsequently in the final image warping and resampling phase of the system. Results using different airborne and spaceborne images were evaluated and showed an average feature correspondence rate of about $80 \%$. 
The technique presented in [9] is based on the combination of feature-based and area-based matching. Least square matching is then employed to refine the process of control point selection. The links of corresponding feature points are established by relaxation techniques based on probability, instead of traditional linking methods based on complicated graphic processing techniques. IKONOS and QuickBird data were used to evaluate the technique.

Sui et. al. [8] presented an algorithm to register remote sensing images with GIS data. The algorithm is based on extracting and recognizing water surfaces in the images and the GIS data. Hence, these surfaces are used to extract bridges. Unchanged features are then extracted and a shape matrix [3] is then established and solved to define corresponding features using the technique presented in [16]. A survey of recent image registration techniques is presented in [18].

Shapiro and Brady [15] devised a method that models the content of each image by means of an intra-image point proximity matrix, and then evaluates the similarity between images by comparing the matrices. The proximity matrices are build using the Gaussian as a weighting function. For each proximity matrix, a modal matrix (a matrix the columns of which are eigenvectors of the original matrix) is built. Each row of the modal matrix represents one point of the corresponding image. The authors find the correspondences by comparing the rows of the two modal matrices, using a binary decision function based on the Euclidean distance.

\section{THE SCOTT AND LONGUET-HIGGINS ALGORITHM}

Scott and Longuet-Higgins proposed a novel and direct method to solve the point correspondence problem between any two arbitrary patterns. The algorithm utilizes a main property of the SVD to satisfy both exclusion and proximity principles. The advantages of the algorithm is its straightforward implementation founded on a well conditioned eigenvector solution which involves no explicit iterations.

The algorithm is described as follows; let $\mathrm{N}$ and $\mathrm{M}$ be two patterns, containing $m$ features $M_{i}(i=1: m)$ and $n$ features $N_{j}(j=1: n)$, respectively. The end result is to find a one-to-one correspondence matrix between the features of the two patterns. The algorithm consists of the following stages:

1. Build a proximity matrix $G$ of the two sets of features where each element $G_{i j}$ is Gaussian-weighted distance between two features $M_{i}$ and $M_{j}$ as shown in equation (1) as presented in [14].

$$
G_{i j}=e^{-r_{i j}^{2} / 2 \sigma^{2}} \quad \mathrm{i}=1,2, \ldots, \mathrm{m} \quad \mathrm{j}=1,2, \ldots, \mathrm{n}
$$

Where $r_{i j}$ is the Euclidean distance between the two features ( $i$ and $j$ ) if they are regarded as lying on the same plane, $\sigma$ controls the degree of interaction between the two sets of features: a small value enforces local interactions, while a larger value permits more global interactions.

2. Perform the SVD of $\mathrm{G}: \mathrm{G}=\mathrm{TDU}^{\mathrm{T}}$

3. Convert $\mathrm{D}$ to a new matrix $\mathrm{E}$ obtained by replacing every diagonal element $\mathrm{D}_{\mathrm{ii}}$ with 1 and then compute the product $\mathrm{P}=\mathrm{TEU}^{\mathrm{T}}$.

4. This new matrix $P$ has the same shape as the proximity matrix $G$ and has the interesting property of sort of "amplifying" good pairings and "attenuating" 
bad ones. Hence, if $P_{i j}$ is both the greatest element in its row and the greatest element in its column, then we regard those two different features $I_{i}$ and $J_{j}$ as being in 1:1 correspondence with one another; if this is not the case, it means that features $I_{i}$ competes unsuccessfully with other features for partnership with.

According to [14], the algorithm is based on the two principles of proximity and exclusion, that is corresponding points must be close, and each point can have one corresponding point at most. The idea is to obtain from the similarity matrix $\mathrm{G}$ a matrix $\mathrm{L}$ such that the entry at row $\mathrm{i}$ and column $\mathrm{j}$ is 1 if $\mathrm{i}$ and $\mathrm{j}$ are corresponding points, 0 otherwise. The matrix P computed by the algorithm is orthogonal, as all the singular values are 1, and it is the orthogonal matrix closest to the proximity G. Following this idea the algorithms establishes a correspondence between the points $i$ and $j$ if the entry $\mathrm{Pij}$ is the largest element in row $\mathrm{i}$ and the largest element in row $\mathrm{j}$.

In the case of real images, the algorithm has been used widely to solve the point correspondence problem between a pair of images [13]. The proximity matrix, in [13] was based on both image intensity and geometry as shown in equation (2). The same algorithm was used in [17] with different formation of the proximity matrix using image correlations and Euclidean distances between image points. In addition, [2] used the another from of the proximity matrix to solve the corresponding problem using scale invariant features presented in [12].

$G_{i j}=\left[e^{-\left(C_{i j}-1\right)^{2}} / 2 \gamma^{2}\right]\left[e^{-r_{i j}^{2} / 2 \sigma^{2}}\right]$

Where $\mathrm{C}_{\mathrm{ij}}$ presents the normalized image intensity correlation between the two images features ( $i$ and $j$ ), $\gamma$ determines how quickly the value of $C_{i j}$ decreases, and other parameters are presented in equation 1.

\section{THE PROXIMITY MATRIX FOR REMOTE SENSING IMAGES}

In this research two different proximity matrices were investigated. The first matrix is based on computing the Euclidean distance between the points in the two satellite images as presented in [14]. In addition, a modified version is introduced and investigate. In this version, the elements $G_{i j}$ of the proximity matrix are computed based on a similarity measure. This measure is based on the 4 parameter transformation model and is computed as follows. Assume two features I and $\mathrm{J}$ in the $1^{\text {st }}$ and $2^{\text {nd }}$ image respectively. Given the coordinates of the start point in each image, the resolution of each image, and the orientations of both images, a similarity equation could be driven between the coordinates of both images as shown in equation $3,[1]$.

$$
\begin{aligned}
& r_{i j}=D_{i j}^{T} \otimes D_{i j} \\
& D_{i j}=\left[\begin{array}{l}
x_{i} \\
y_{i}
\end{array}\right]-\mathrm{S}\left[\begin{array}{cc}
\cos \theta & -\sin \theta \\
\sin \theta & \cos \theta
\end{array}\right]\left[\begin{array}{l}
u_{j} \\
v_{j}
\end{array}\right]+\left[\begin{array}{l}
t_{x} \\
t_{y}
\end{array}\right]
\end{aligned}
$$


Where $S$ is the scale factor between the two images, $\theta$ is the rotation angle between the two images, $t_{x}$ and $t_{y}$ are shift values between the two images, $x_{i}$ and $y_{i}$ are the coordinates of the (i) point in the first image, and $u_{j}$ and $v_{j}$ are the coordinates of the $(j)$ point in the second image.

The value of $r_{i j}$ is then used to fill the proximity matrix. Several reasons stand behind using this form. First, the scale differences between the satellite images are usually larger than the scale differences between normal close range images. Another reason is that the orientation and other geometric parameters are usually affordable in the header files. Even if the header files are not available these parameters could be computed easily given the approximate orientations of the satellite images. The scale (S) can be computed as the ratio between the resolutions of the images. The translation $\left(t_{x}\right.$ and $\left.t_{y}\right)$ could be computed as the differences between the image coordinates of a given point. The rotation angle between the two images could be approximately measured given the coordinates of two points. Hence, these approximate values could be used as the initial values for the computation of the condition equation of the 4 parameter transformation model with no need to the exact values.

\section{DATASET DESCRIPTION}

The dataset used in this research consists of three satellite images covering West Lafayette city, IN. The first image is an SPOT4 panchromatic image, with a pixel size of 10 meters. The second image is an IKONOS panchromatic image, with a pixel size of 1 meter. The third image is an QuickBird multispectral image, with a pixel size of 2.4 meters. Forty points were randomly digitized in the three images and there coordinates were recorded in each image. Figure 1, 2, and 3 show the point distribution over the IKONOS, QuickBird, and SPOT images respectively.

\section{EXPERIMENTAL RESULTS AND ANALYSIS}

Three experiments are conducted to find the corresponding point pairs between each two images. In the first experiment, the IKONOS and the QuickBird images are used. In the second experiment, the IKONOS and the SPOT images are used. In the last experiment, the QuickBird and the SPOT images are used. For each experiment, two proximity matrices, using the Euclidean distance and the 4 parameter transformation, are tested. Different values for the $\sigma$ ranging from 0.05 to 1.0 are tested. In the implementation steps, the values of the proximity matrices were normalized by dividing by the maximum proximity cost. For the 4 parameters tests, approximate values for the transformation parameters are used to find the correspondence points. After finding the correct correspondent points in the images, the least squares adjustment technique was used to find the adjusted values of the transformation parameters. Table 1 shows the approximate and adjusted transformation parameters. 


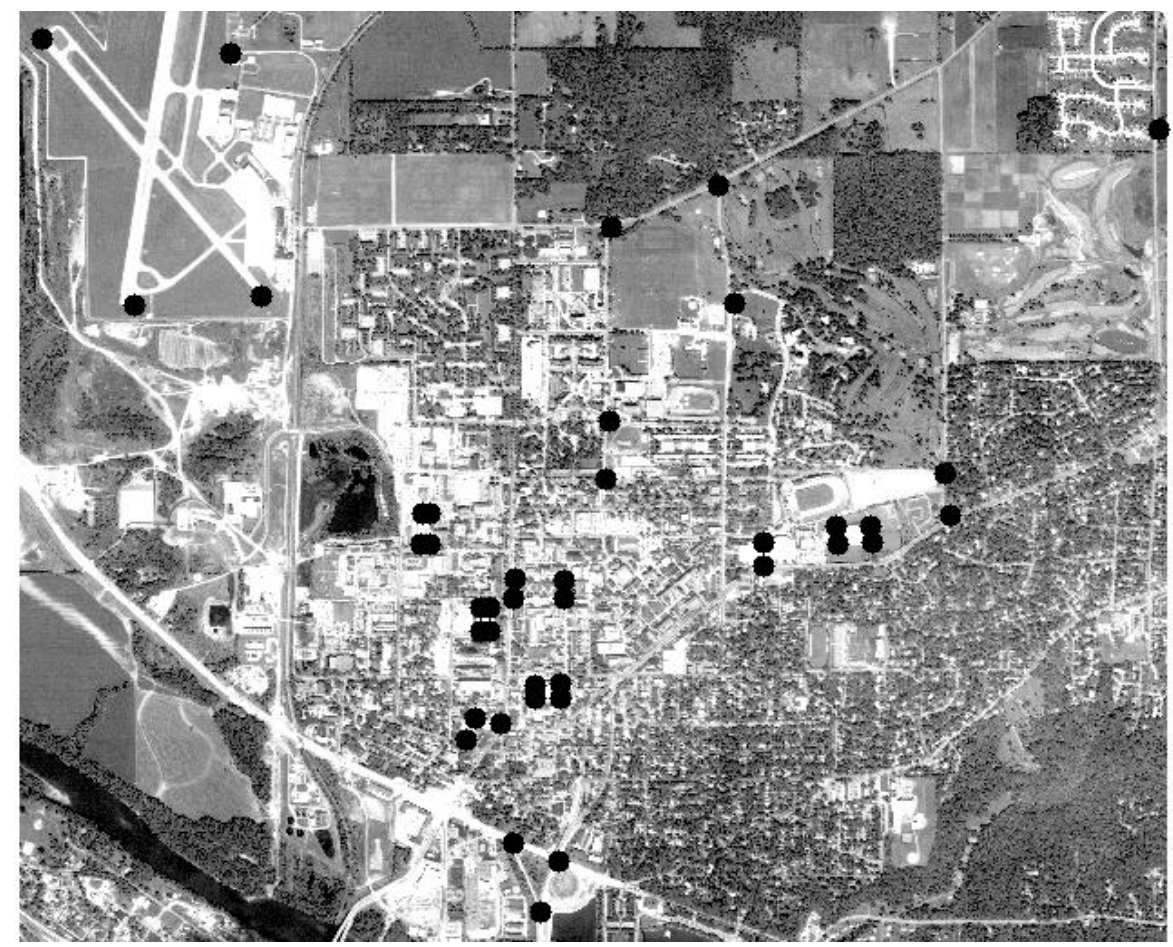

Figure 1. Point distribution over the IKONOS image

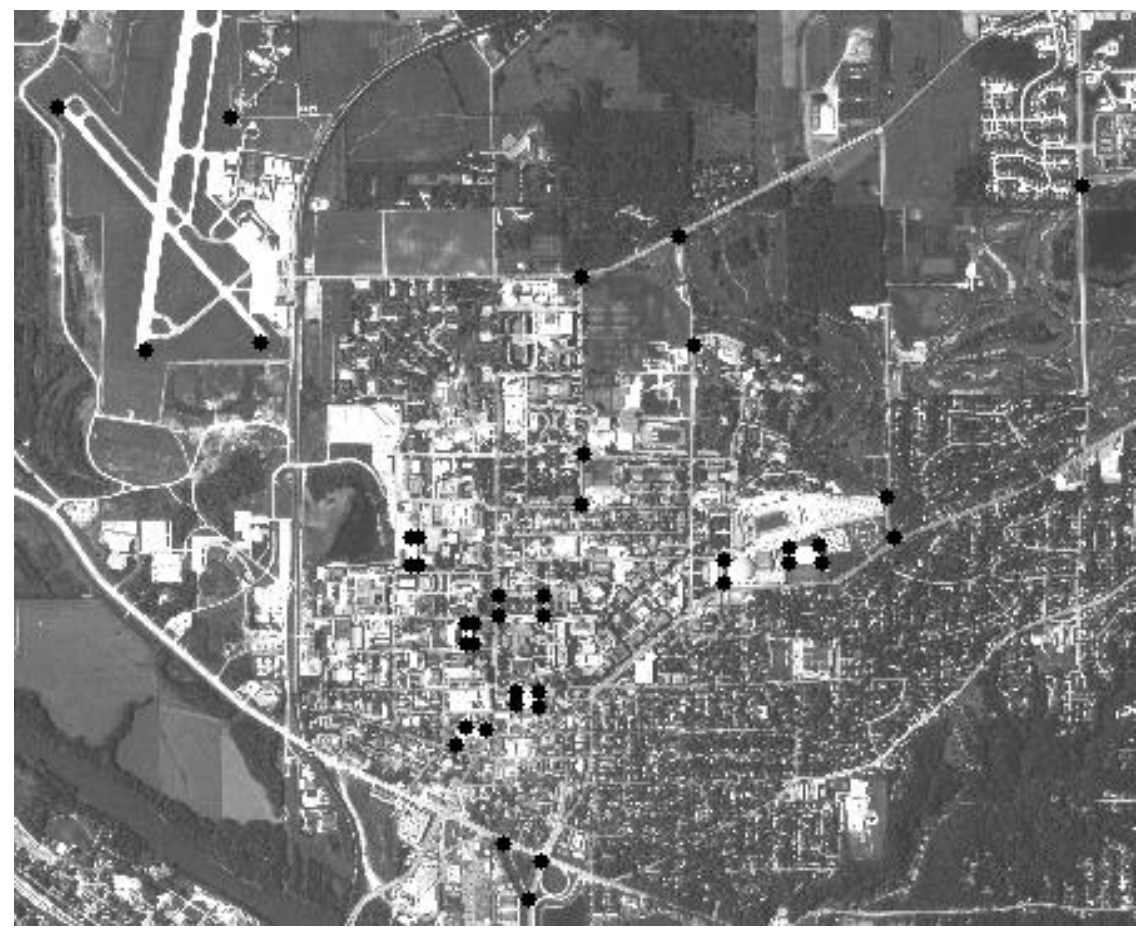

Figure 2. Point distribution over the QuickBird image 


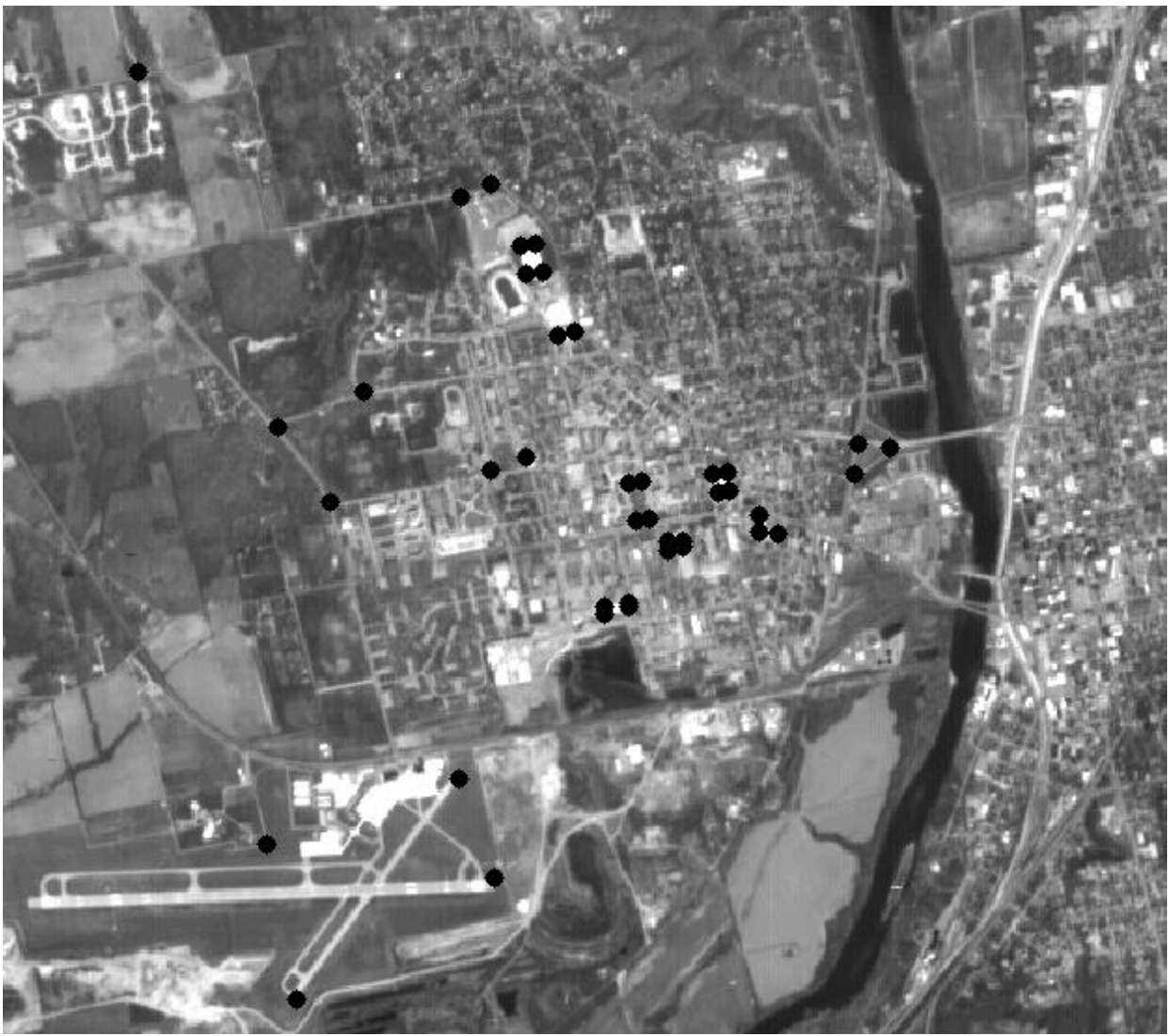

Figure 3. Point distribution over the SPOT image

Table 1. Transformation parameters between the SPOT, IKONOS, and QuickBird satellite images

\begin{tabular}{|c|c|c|c|c|}
\hline \multicolumn{2}{|c|}{} & $\begin{array}{c}\text { SPOT- } \\
\text { IKONOS }\end{array}$ & $\begin{array}{c}\text { SPOT- } \\
\text { QuciBird }\end{array}$ & $\begin{array}{c}\text { IKONOS- } \\
\text { QuickBird }\end{array}$ \\
\hline \multirow{2}{*}{$\mathrm{S}$} & Approx. & 10 & 4 & 0.4 \\
\cline { 2 - 5 } & Adjusted & 9.9738 & 3.9374 & 0.3954 \\
\hline \multirow{2}{*}{$\theta^{\circ}$} & Approx. & 13 & 12.2 & -0.8 \\
\cline { 2 - 5 } & Adjusted & 14.3912 & 13.8910 & -0.3211 \\
\hline \multirow{2}{*}{$\mathrm{t}_{\mathrm{x}}$ (pixels) } & Approx. & 3589 & 3870 & 281 \\
\cline { 2 - 5 } & Adjusted & 3588.13 & 3868.1 & 281.03 \\
\hline \multirow{2}{*}{$\mathrm{t}_{\mathrm{y}}$ (pixels) } & Approx. & 759 & 1872 & 1068 \\
\cline { 2 - 5 } & Adjusted & 762.38 & 18879.68 & 1073.41 \\
\hline
\end{tabular}

Results are represented in figures 4 and 5. The figures show that the Euclidean distance results are not stable. In addition, the results of the Euclidian distance showed that for $(\sigma<0.2)$ the percentage of correctly matched points is low. This is due to the geometric characteristics of the images. However, the 4 parameters results are stable. Moreover, the percentage of correctly matched points is about $100 \%$ for $(\sigma>0.05)$ and 
$(\sigma<0.5)$. Results for the 4 parameters also showed that the percentage of correctly matched points is independent of the values of the transformation parameters.

\section{CONCLUSIONS}

This paper presented a modified method to solve the point correspondence problem between different satellite images. The paper is based on the Scott and LonguetHiggins algorithm, used to solve the point correspondence problem between close range images. Two different proximity matrices are examined. The research showed that the Euclidean proximity matrix may not successfully be used to solve the point correspondence problem between satellite images. This is due to the rigorous transformation between the images. Satellite images usually don't have the same resolution nor orientation. Hence, a modified proximity matrix was introduced to overcome these problems. This matrix is based on the 4 parameter transformation. Results showed that the modified matrix provides a comprehensive solution for the point correspondence problem between satellite images. The new version is stable regardless of the values of $\sigma$, the orientation of the satellite images, the scale ratio between the images, or the translation values between the images. Hence, the presented method could be used to solve the point correspondence problem between any pair of satellite images. This will provide high quality inputs for a variety of applications including change detection applications. Future work will focus on using the other correlation measures such as the surrounding intensities. In addition, testing the proposed algorithm with other datasets. The effect of the control point distribution on the qulity of the results will also be addressed.

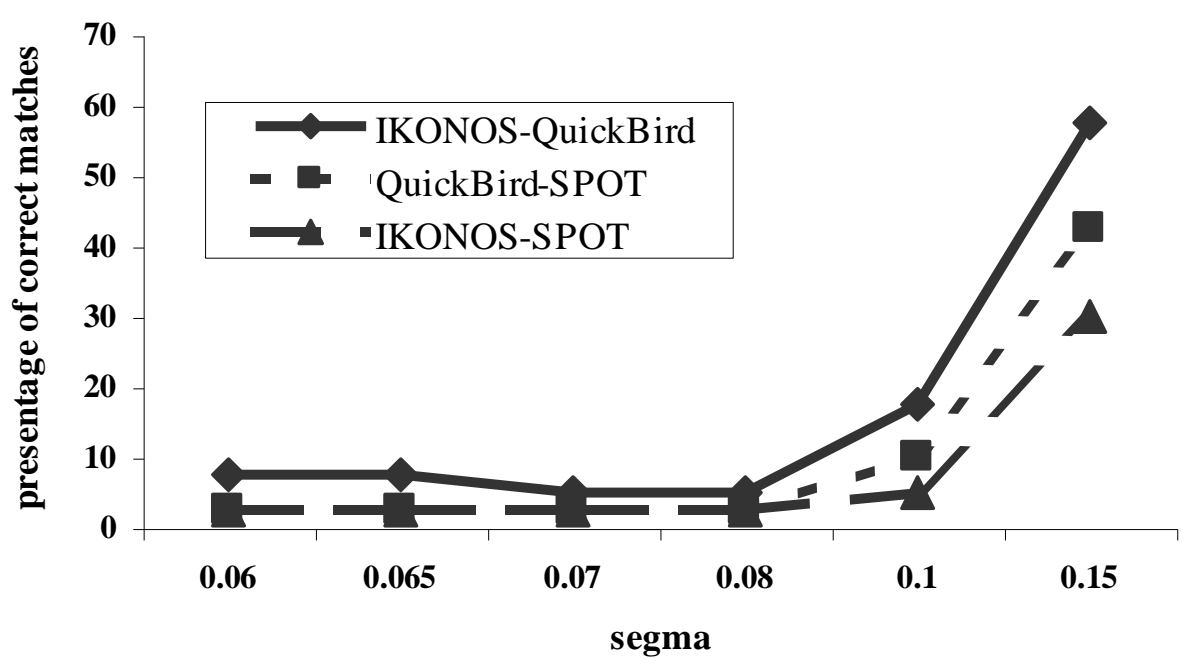

Figure 4. Percentage of points correctly matched using the Euclidean distance measure 


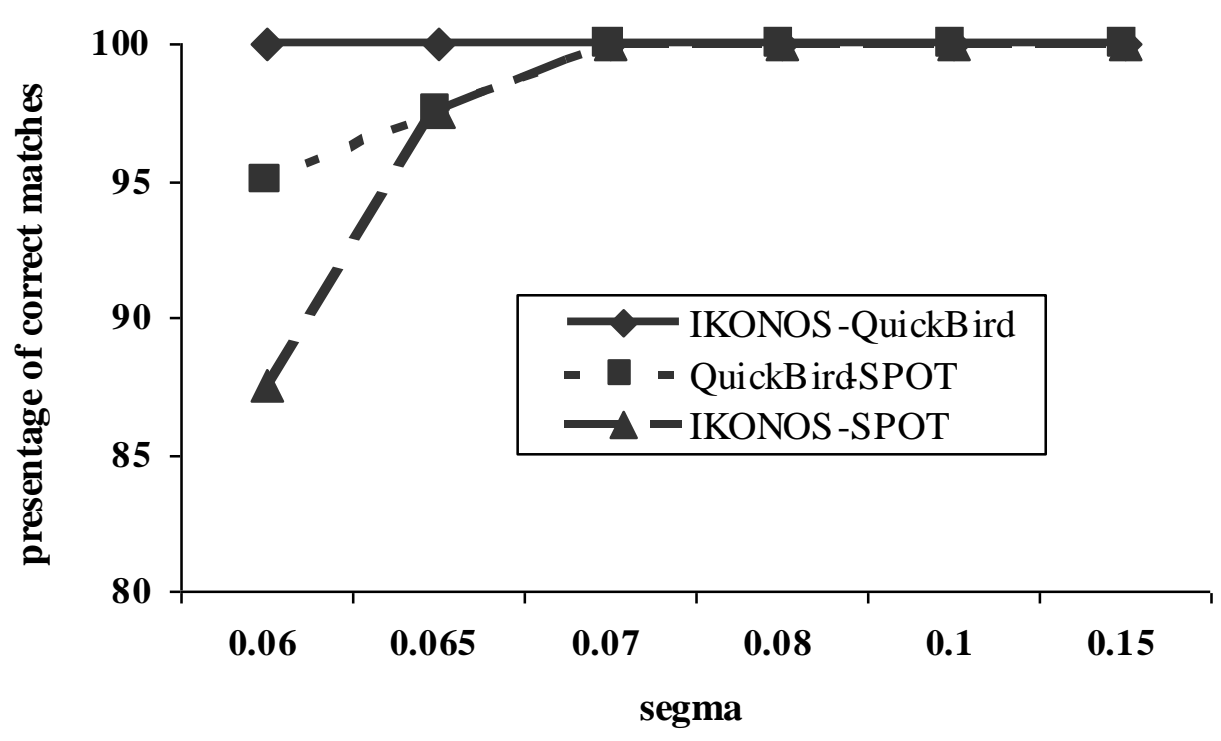

Figure 5. Percentage of points correctly matched using the 4 parameters transformation measure

\section{REFERENCES}

1. Al-Razuq, R., "Semi-Automatic Registration of Multi-Source Satellite Imagery with Varying Geometric Resolutions", $\mathrm{PhD}$ thesis, Calgary University, Calgary, Alberta, 2004.

2. Delponte, E., Isgre, F., Odone, F., and Verri, A., "SVD-matching using SIFT Features", Proceedings of the International Conference on Vision, Video and Graphics, Edinburgh, UK, 7 -8, July, 2005.

3. Flusser, J., "Invariant Shape Matrix Description and Measure of Object Similarity", International Proceedings Of the $4^{\text {th }}$ International Conference on Image Processing and its Applications, Maastricht, pp. 139 - 142, 7 - 9 April, 1992.

4. Habib A., and R. Al-Ruzouq, "Semi-Automatic Registration and Change Detection Using Multisource Imagery with Varying Geometric and Radiometric Properties", Photogrammetric Engineering and Remote Sensing, 71(3): 325-332, March, 2005.

5. Habib, A., 2000, "New Approach to Solving Matching Problems in Photogrammetry", Proceedings of the International Archives of Photogrammetry and Remote Sensing, Amsterdam, 31(B2): 257 - 264, Amsterdam, The Netherlands, 16 - 22 July, 2000.

6. Habib, A., and D. Kelley, "Automatic Relative Orientation of Large Scale Imagery over Urban Areas using Modified Iterated Hough Transform", International Journal of Photogrammetry and Remote Sensing, 56: 29-41, 2001.

7. Habib, A., R. Al-Ruzouq, and C. J. Kim, "Semi-Automatic Registration and Change Detection Using Multisource Imagery with Varying Geometric and 
Radiometric Properties", Proc. of the International Archives of Photogrammetry and Remote Sensing, Istanbul, Turkey, Vol. 35 (B2): 445 450, 12 -23 July, 2004.

8. Haigang, S., Jianya, G., Jun, X., and Li, M., "Automatic Recognition of Bridges over Water and Registration in Remotely Sensed Images with GIS Data", Proceedings of the ISPRS conference, Remote Sensing: From Pixels to Processes, Enschede, The Netherlands, 8-11 May, 2006.

9. Hong, G, Y., Zhang, and D., Fraser, “ A New Image Registration Technique in Urban Change Detection", Proceeding of the 2004 ASPRS Annual Conference, Denver, USA, 23 - 28 May, 2004.

10. Lavigne, D., "AMIR: A New Automated Multisensor Image Registration System", Proceeding of the 2006 ASPRS Annual Reno, Nevada, USA, 1 - 5 May, 2006.

11. Li, D., S. Haigang, and X. Ping, "Automatic Change Detection of Geospatial Data from Imagery", Proceeding of the ISPRS Conference titled Integrated System for Spatial Data Production, pp. 245-252, Xi'an, China, 20-23 August, 2002.

12. Lowe, D.G., "Distinctive Features from Scale-invariant Keypoints", International Journal of Computer Vision, 60(2): 91-110, 2004.

13. Pilu, M., "Uncalibrated Stereo Correspondence by Singular Value Decomposition", Proceedings of the Computer Vision \& Pattern Recognition Conference, Puerto Rico, USA, pp. 261 - 266, 17-19 June, 1997.15.

14. Scott, G., and Longuet-Higgins, H., C., "An Algorithm for Associating the Features of Two Images", Proceedings of the Royal Society London, B244, pp. 21-26.

15. Shapiro, L. S., and J., M., Brady, "Feature-based correspondence - an eigenvector approach", Image Vision Computing, 10, 1992.

16. Zhang, Z.H., "An Automatic Procedure for SAR-optical Satellite Image Registration based on Multi-layer Feature Matching Strategy", Intelligent Signal Processing and Communication Systems, ISPACS, Proceedings of 2004 International Symposium, pp. 574 -580, Seoul, Korea, 18 - 19 November, 2004.

17. Zhao, F., "Image Matching Based on Singular Value Decomposition", $5^{\text {th }}$ Pacific Rim Conference on Multimedia, Tokyo, Japan, pp. 119-126, 30 Nov. - 3 Dec., 2004.

18. Zitova, B., and Flusser, J., "Image Registration Methods: A Survey". Image and Vision Computing, 21: 977-1000, 2003. 
حل مشكلة النقاط المتناظرة بين صور الأقمار الصناعية باستخدام نسخة معدلة من

\section{طريقة تحليل القيم الأحادية لإيجاد النقط المتناظرة}

تعتبر صور الأقمار الصناعية المصدر الرئيسي لعدد من التطبيقات في مجالات التعرف على التغيرات الطبيعية والجيولوجية والطبوغرافية. ولكن هذه الصور في العادة تكون متعددة وتم التقاطها في أوقات لعنات مختلفة. ولذا فأن النقاط المناظرة تكون غير محددة. هذا البحث بيتخدم طريقة تحليل القيم الأحادية لحل مشكلة إيجاد النقاط المناظرة بين صور الأقمار الصناعية ذات الخواص المختلفة آليا. ويتم في هذه العملية بناء مصفوفة تكلفة بين كل زوج من النقاط في صورتين. وعناصر هذه المصفوفة لئن

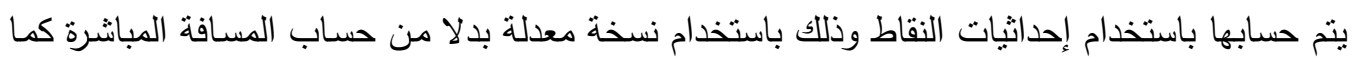

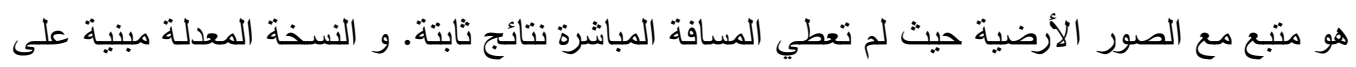
الخواص الهندية للصور ـ وقد أوضحت نتائج استخدام الطريقة المعدلة تقدم كبير في إيجاد النقاط المتتاظرة في صور الأقمار الصناعية بغض النظر عن حجم ودقة الصور المستخدمة. 